\title{
Divergent pathway of lipid profile components for cardiovascular disease and mortality events: Results of over a decade follow-up among Iranian population
}

Zahra Ghasemzadeh ${ }^{1 \dagger}$, Hengameh Abdii ${ }^{2 \dagger}$, Samaneh Asgari ${ }^{1}$, Maryam Tohidi ${ }^{1}$, Davood Khalili' ${ }^{1}$ Majid Valizadeh ${ }^{3}$, Siamak Moeini ${ }^{1}$, Vahid Eidkhani ${ }^{1}$, Fereidoun Azizi ${ }^{2}$ and Farzad Hadaegh ${ }^{1 *}$

\begin{abstract}
Background: Data regarding the impact of different lipid measures on cardiovascular diseases (CVD) and mortality events is not consistent. We aimed to evaluate the relationship between different lipid parameters and incident CVD and mortality events in an Iranian population over a median follow-up of 11.9 years.

Methods: The study was conducted on 2532 men and 2986 women aged $\geq 40$ years. Multivariate adjusted hazard ratios (HRs), using age as time scale, were calculated for every 1 standard deviation (SD) increase in total cholesterol (TC), logarithm-transformed triglycerides (In-TGs), low density lipoprotein-cholesterol (LDL-C), high density lipoprotein-cholesterol (HDL-C), non-HDL-C, TC/HDL-C and In-TGs/HDL-C. Covariates included gender (female as reference), body mass index, education status, low physical activity, smoking, blood pressure status (normotension, prehypertension and hypertension), glucose tolerance status (normal glucose tolerance, prediabetes and diabetes) and lipid lowering drugs. The same analyses were also repeated for tertiles of all lipid measures. Considering the absence of interaction between gender and lipid parameters, we used a sex-adjusted analysis. For analyses of mortality events, prevalent CVD was adjusted as well (All p for interactions $>0.1$ ).
\end{abstract}

Results: A total of 789 new CVD events, 279 cardiovascular (CV) and 270 non-CV deaths occurred. In multivariate analysis, all lipid measures except HDL-C showed significant risk for new CVD events with HRs ranged from 1.14 to 1.27 for In-TGs/HDL-C and LDL-C, respectively (all $p$-values $\leq 0.001$ ). Considering CV mortality, there were significant positive associations between TC, LDL-C, non-HDL-C, TC/HDL-C and CV mortality events in sex-adjusted analysis; however after multivariate analysis, these associations attenuated and reached to null. Applying lipid measures as categorical variables, only TC displayed a positive association with CV mortality in multivariate analysis [TC $\geq 6.14 \mathrm{mmol} / \mathrm{L}:$ HR $1.43(1.04-1.98)]$.

In multivariate analysis, there were negative significant associations between all lipid measures except HDL-C and non-CV mortality; every 1-SD increase in TC, LDL-C, non-HDL-C, In-TGs, TC/HDL-C and In-TGs/HDL-C was associated with 24, 25, 27, 19, 23 and $17 \%$ decreased risk in non-CV mortality (all $p$-values $\leq 0.01$ ).

Conclusions: These findings indicate divergent associations of TC, LDL-C, non-HDL-C, TC/HDL-C, TGs and TGs/HDL-C with CVD vs non-CV mortality, demonstrating a higher risk for the former and lower risk for the latter.

Keywords: Cardiovascular disease, Mortality, Lipids, Cholesterol, Triglycerides

\footnotetext{
* Correspondence: fzhadaegh@endocrine.ac.ir

${ }^{\dagger}$ Equal contributors

${ }^{1}$ Prevention of Metabolic Disorders Research Center, Research Institute for Endocrine Sciences, Shahid Beheshti University of Medical Sciences, P.O. Box: 19395-476, Tehran, Iran

Full list of author information is available at the end of the article
} 


\section{Background}

Cardiovascular disease (CVD) is the leading cause of $30 \%$ of mortality worldwide [1] and up to $50 \%$ of deaths in Iran [2]. The association between different lipid measures and cardiovascular disease and mortality have been shown in several studies [3-5]. Based on the results of observational and interventional studies conducted in middle-aged populations, there is a continuous graded relationship between serum total cholesterol (TC) levels with CVD and mortality [6]. Meanwhile, non-high density lipoprotein cholesterol (non-HDL-C) as the masses of cholesterol in the atherogenic apo $B$ lipoprotein particles has been known as a valuable predictor for cardiovascular risk [3]. In a cohort of Western population, triglycerides (TGs) and the TC to HDL-C ratio (TC/HDL-C) were more strongly associated with risk of future coronary heart disease (CHD) [7]. Moreover, a recent meta-analysis showed significant association between serum triglycerides (TGs) and CVD and all-cause mortality [5]. There are several studies regarding inverse relationship between HDL-C levels and CVD [8]. Furthermore, many studies have shown that indices such as TGs to HDL-C ratio (TG/HDL-C) and TC/HDL-C can also predict CVD and CHD mortality [4, 9-11]. Despite these, data regarding association between lipid measures and mortality is not consistent $[12,13]$ and to the best of our knowledge, few studies have examined this relationship considering cardiovascular $(\mathrm{CV})$ vs non-CV mortality, separately.

Almost all previous surveys regarding the association between lipid measures and CVD or all-cause mortality events have been performed on European, American or the Far East populations [14, 15] and it is not clear whether their results can be extrapolated to MiddleEastern population who have high incidence of CHD and mortality events [16, 17]. Recently, a multivariate sex-adjusted analysis showed that among Iranian adults aged $\geq 50$ years, serum levels of TC, TGs, low density lipoprotein-cholesterol (LDL-C), non-HDL-C, and TC/ HDL-C were significantly associated with higher risk of incident $\mathrm{CHD}$, whereas HDL-C was associated with a lower risk [18].

In the current population-based study, we expand our previous findings and investigate the association between seven lipoprotein measures and CVD events, CV and non-CV mortality in more than a decade follow-up.

\section{Methods}

\section{Study population}

Subjects for the current study were selected from participants of the Tehran Lipid and Glucose Study (TLGS), a long term prospective population-based study being conducted on a representative sample of Tehranian residents to determine the prevalence and incidence of non- communicable diseases and related risk factors. Details of TLGS have been published previously [19]. TLGS has two main phases: a cross-sectional phase (1999-2001) and a prospective ongoing phase consisting of follow-up visits at 3-year intervals.

A total of 6445 individuals, aged $\geq 40$ years were enrolled in the first $(n=5406)$ or second $(n=1039)$ phases. To evaluate the effects of lipid markers on CVD and non-CV mortality, after excluding participants without any follow-up $(n=618)$ or with missing data for baseline covariates $(n=309), 5518$ participants with complete data were followed for a median of 11.9 years (interquartile range: 7.8-13.1 years) up to March 2012. For investigating the effects of lipid measures on CVD events, the analysis was done in a smaller sample $(n=5054)$ by excluding individuals with prevalent CVD.

During the third phase of the TLGS (2006-2008), a total of 4920 participants were randomly selected for completing the dietary assessment based on their age and sex. Finally, the dietary data for 3462 subjects were completed using a validated 168-item food frequency questionnaire [20]. For the present study, after exclusion of participants aged $<40$ years and those with under- or over-reporting of dietary energy intakes (<800 or $\geq 4200$ kilocalories/day), dietary information of 915 participants was included in a sensitivity analysis. Furthermore, data regarding serum insulin was available for 1548 participants at baseline which was considered in a subgroup analysis.

The ethics committee of the Research Institute for Endocrine Sciences, Shahid Beheshti University of Medical Sciences approved the design of TLGS and all participants provided written informed consent.

\section{Data collection}

\section{Medical history, clinical examination and laboratory measurements}

Participants were interviewed face to face by trained interviewers. A standard questionnaire was used to collect demographic information, including their smoking status and taking of any antidiabetic or hypertension drugs. Systolic blood pressure (SBP) and diastolic blood pressure (DBP) were measured twice in a seating position on the right arm, using a standard mercury sphygmomanometer and the mean value was considered as the subject's SBP and DBP.

Weight was measured with individuals minimally clothed without shoes, using digital scales (Seca 707: range $0.1-150 \mathrm{~kg}$ ) and recorded to the nearest $0.1 \mathrm{~kg}$. Height was measured in a standing position without shoes, using a tape meter, while shoulders were in a normal alignment. Body mass index (BMI) was calculated as weight in kilograms divided by height in square meters. Waist circumference (WC) was measured at the level of umbilicus. 
Venous samples were collected in vacationer tubes after 12-14 h overnight fasting between 7:00 and 9:00 A.M and centrifuged within 30-45 min of collection. Fasting plasma glucose (FPG) and 2-h post challenge plasma glucose (2 h-PCPG) were measured by the enzymatic colorimetric method, using glucose oxidase; inter- and intra-assay coefficients of variation at baseline and follow-up phases were both less than $2.3 \%$. TC was assayed, using the enzymatic colorimetric method with cholesterol esterase and cholesterol oxidase. HDL-C was measured after precipitation of the apolipoprotein $B$ (apo B)-containing lipoproteins with phosphotungistic acid. TGs were assayed using glycerol phosphate oxidase. Both intra- and inter-assay coefficients of variations for TC, HDL-C and TGs were less than 1.9, 3 and $2.1 \%$, respectively. Analyses were performed using related kits (Pars Azmon Inc., Tehran, Iran) and a Selecta 2 autoanalyzer (Vital Scientific, Spankeren, Netherlands) on the day of blood sampling. All assays were done when quality control met the acceptable criteria. To calculate LDL-C, a modified Friedewald equation was used [21].

\section{Definition of terms}

Participants were classified as having type 2 diabetes if they met at least one of the following criteria: FPG $\geq$ $7 \mathrm{mmol} / \mathrm{L}, 2 \mathrm{~h}-\mathrm{PCPG} \geq 11.1 \mathrm{mmol} / \mathrm{L}$ or taking antidiabetic medications. Moreover, prediabetes was defined as having a $5.55 \mathrm{mmol} / \mathrm{L} \leq \mathrm{FPG}<7 \mathrm{mmol} / \mathrm{L}$ and/or a $7.77 \mathrm{mmol} / \mathrm{L} \leq 2 \mathrm{~h}-\mathrm{PCPG}<11.1 \mathrm{mmol} / \mathrm{L}$, without using glucose lowering drugs; those with $\mathrm{FPG}<5.55 \mathrm{mmol} / \mathrm{L}$ and $2 \mathrm{~h}$-PCPG $<7.77 \mathrm{mmol} / \mathrm{L}$ were considered as normal glucose tolerant according to the definition of American Diabetes Association [22]. Hypertension was defined as systolic blood pressure (SBP) $\geq 140 \mathrm{mmHg}$ or diastolic blood pressure (DBP) $\geq 90 \mathrm{mmHg}$, or taking antihypertensive medications. Prehypertension was defined as $\mathrm{SBP} \geq 120 \mathrm{mmHg}$ and $<140 \mathrm{mmHg}$ and $\mathrm{DBP} \geq 80 \mathrm{mmHg}$ and $<90 \mathrm{mmHg}$ and normotension as $\mathrm{SBP}<120 \mathrm{mmHg}$ and $\mathrm{DBP}<80 \mathrm{mmHg}$ without any medication use [23]. A current smoker was defined as a person who smokes cigarettes daily or occasionally. Low physical activity was defined as vigorous activity less than three times per week for participants entered in first phase and as metabolic equivalent task-minutes per week $<600$ for those who entered in the second phase [24]. Education status was categorized into 3 groups: < 6 years, 6-12 years and $\geq 12$ years. With respect to the Kidney Disease Outcome Quality Initiative guidelines, chronic kidney disease (CKD) was defined as either kidney damage or estimated Glomerular Filtration Rate $(\mathrm{eGFR})<60 \mathrm{~mL} / \mathrm{min} / 1.73 \mathrm{~m}^{2}$ for $>3$ months [25].

\section{Outcome measurement}

Details of cardiovascular outcomes have been published previously [19]. In the ongoing TLGS, all participants are followed up for any medical event during the previous year by telephone. They are questioned by a trained nurse regarding any medical conditions or whether a related event had occurred; a trained physician collected complementary data during a home visit and/or a visit to the respective hospital to collect data from medical files; in case of mortality, data are collected from the hospital or death certificate by an authorized local physician, then evaluated by an outcome committee consisting of a principal investigator, an internist, an endocrinologist, a cardiologist, an epidemiologist and the physician who collects the outcome data. Other experts are invited for evaluation of non-communicable diseases, as needed. A specific outcome for each event is assigned, based on the International Statistical Classification of Diseases and Related Health Problems criteria, 10th Revision, and the American Heart Association classification for cardiovascular events $[19,26]$. In this study, first fatal and non-fatal CHD event, stroke or cerebrovascular accidents were considered CVD events. By definition, CHD includes cases of definite myocardial infarction diagnosed by electrocardiogram (ECG) and biomarkers, probable myocardial infarction (positive ECG findings plus cardiac symptoms or signs and biomarkers showing negative or equivocal results), unstable angina pectoris (new cardiac symptoms or changing symptom patterns and positive ECG findings with normal biomarkers) and angiographic proven CHD. CV mortality is specified as a composite measure of any fatal CVD events, including fatal CHD and fatal stroke.

\section{Statistical analysis}

Mean and standard deviation (SD) values for continuous and frequencies (\%) for categorical variables of the baseline characteristics were described and median (interquartile range) for TGs and TGs/HDL-C as they had skewed distribution.

Cox proportional hazard models with age as time scale were used to evaluate associations of CVD events, CV and non-CV mortality with one standard deviation (1$\mathrm{SD})$ change in TC, LDL-C, HDL-C, non-HDL-C as well as 1-SD increase in TC/HDL-C [27]. For TGs and TGs/ HDL-C, hazard ratios (HRs) were calculated for every 1 SD increase in logarithm (ln)-transformed form of these measures. The event time was defined as the time between entrance to the study and the endpoints. Endpoints were considered as CVD event, $\mathrm{CV}$ and non-CV mortality. Also censored data was defined as individuals either lost to follow-up, having left residential area, having non-CV mortality (for CV mortality endpoint) or continued up to March 2012, whichever occurred earlier.

Variables with $p$-values $<0.2$ in univariate analysis were included in the multivariate Cox model. Interaction between gender and lipid measures with CVD event, CV and non-CV mortality was checked by log-likelihood 
ratio test, in multivariate analysis; since, there was no significant interaction (all $p$-values $>0.1$ ), analysis was repeated in a pooled sample to reach acceptable statistical power. Also, the effect of lipid measures on CVD events was analysed in both genders. The interaction between prevalent CVD and lipid lowering drugs with lipid markers was checked and since no significant interactions were found (all $p$-values $>0.1$ ), they were adjusted in multivariate model.

In multivariate analysis, two models were applied; model 1 included baseline levels of lipid profiles and gender (female as reference); model 2, further adjusted for potential covariates, included BMI, education status ( $\geq 12$ years as reference group), low physical activity, smoking, blood pressure status (normotension as reference, prehypertension, hypertension), glucose tolerance status (normal glucose tolerance as reference, prediabetes, diabetes), lipid lowering drugs, and prevalent CVD (for $\mathrm{CV}$ and non-CV mortality events). Additionally, analyses were repeated for tertiles of all lipid measures with the same approach. The annual $\mathrm{CV}$ and non-CV mortality rate (per 1000 person-year) for each tertile of lipid-measures was calculated by dividing the total number of incident events to the total person-years. The proportionality of the multivariable Cox model was assessed using Schoenfeld's global test of residuals $(p>0.1)$.

Dietary intakes of nutrients were adjusted for total energy intakes, according to residuals method [28]. Potential confounding effects of homeostatic model assessmentinsulin resistance (HOMA-IR) and dietary factors including total fat, dietary cholesterol, total fiber, and $\mathrm{Na} / \mathrm{K}$ ratio were assessed for sensitivity analysis.

Statistical analyses were performed using STATA version 12.0 (Stata Corp LP, College Station, Texas). $P$ values $<0.05$ were considered statistically significant.

\section{Results}

The study sample consists of 5518 individuals (2532 men and 2986 women), with mean age (SD) of 55.46 (10.73) years in men and 53.18 (9.36) years in women. Baseline characteristics in men and women are shown in Table 1 . There were significant difference in all variables between men and women, except for TGs. Men were older and had lower BMI, TC, LDL-C, HDL-C as well as lower prevalence of prediabetes, diabetes, hypertension and CKD; however, the levels of non-HDL-C, TC/HDLC, ln-TGs/HDL-C, prevalence of CVD, rate of current smoking, prehypertension and low levels of physical activity were higher in men.

We ascertained 789 incident CVD events among participants free of CVD at baseline $(n=5054)$. The incidence of CVD event for each 1000 person-year is shown in Additional file 1: Table S1. In total population, the multivariate adjusted HRs of CVD events for every 1-SD increase in TC, LDL-C, non-HDL-C, ln-TGs, TC/HDL$\mathrm{C}$ and $\ln$-TGs/HDL-C were associated with $26,27,22$, 15,18 and $14 \%$, respectively (Table 2); however, the corresponding change in HDL-C was associated with $7 \%$ decreased risk of CVD, which did not reach to statistical significance $(p=0.07)$. Sex-stratified analysis did not change the association between lipid parameters and CVD events. Analysis using tertiles of lipid measures for incident CVD showed the same results (Table 3).

During the study period, among the whole population, 549 mortality events (341 men and 208 women) comprising $279 \mathrm{CV}$ mortality (183 men and 96 women) and 270 non-CV mortality (158 men and 112 women) events, occurred. Different causes of non-CV mortality included cancer $(n=85)$, sepsis $(n=46)$, accidents $(n=$ 16), chronic obstructive pulmonary diseases $(n=2)$, and unknown or miscellaneous causes $(n=121)$. The incidence of $\mathrm{CV}$ and non-CV mortality for each 1000 person-year is shown in Additional file 1: Tables S2 and S3.

There were significant positive associations between TC, LDL-C, non HDL-C and TC/HDL-C and CV mortality events in sex-adjusted analysis (model 1); however after adjustment with different covariates, these associations attenuated and reached to null (Table 4). Applying lipid measures as categorical rather than continuous variables, TC $\geq 6.14 \mathrm{mmol} / \mathrm{L}$ was associated with $52 \%$ and $43 \%$ increase in CV mortality risk in model 1 and 2, respectively (Table 5). As shown in Table 6, in sex- and multivariate adjusted analyses, there were negative significant associations between all lipid measures except HDL-C and non-CV mortality so that every $1-\mathrm{SD}$ increase in TC, LDL-C, non-HDL-C, ln-TGs, TC/HDL-C and $\ln$-TGs/HDL-C were associated with 24, 25, 27, 19 , 23 and $17 \%$ decrease in non-CV mortality. When lipid measures were applied as categorical variables, the results generally remained unchanged (Table 7). In a sensitivity analysis, excluding individuals who died within 3 years after initiation of the study as well as those with $\mathrm{BMI} \leq 18.5 \mathrm{~kg} / \mathrm{m}^{2} \quad(n=164)$, the association between lipid parameters and mortality events did not change (Data not shown).

The association between lipid measures and CVD events among 1548 participants, free of prevalent CVD at baseline and available data on HOMA-IR, is shown in Additional file 1: Tables S4 and S5. There was significant association between lipid parameters and CVD events only in sex-adjusted analysis; however, applying lipid measures as categorical variables, significant risk for CVD events was displayed in the second and third tertiles of TC, LDL-C and non-HDL-C also third tertile of TC/HDL-C.

\section{Discussion}

This study determined relationship between different lipid measures with CVD, CV and non-CV mortality 
Table 1 Baseline characteristics of participants in men, women and total population; Tehran Lipid and Glucose Study (TLGS) (2001-2012)

\begin{tabular}{|c|c|c|c|c|}
\hline & Total $(N=5518)$ & Men $(N=2532)$ & Women $(N=2986)$ & $p$-value \\
\hline Age, years & $54.23(10.07)$ & $55.46(10.73)$ & $53.18(9.36)$ & $<0.001$ \\
\hline $\mathrm{BMl}, \mathrm{kg} / \mathrm{m} 2$ & $27.86(4.59)$ & $26.24(3.89)$ & 29.24(4.69) & $<0.001$ \\
\hline$W C, \mathrm{~cm}$ & $92.78(11.17)$ & $91.26(10.5)$ & $93.46(11.42)$ & $<0.001$ \\
\hline $\mathrm{SBP}, \mathrm{mm} \mathrm{Hg}$ & 126.78(21.16) & 125.96(20.8) & $127.47(21.45)$ & 0.008 \\
\hline $\mathrm{DBP}, \mathrm{mm} \mathrm{Hg}$ & $80.13(11.49)$ & 79.35(11.88) & $80.8(11.10)$ & $<0.001$ \\
\hline $\mathrm{FPG}, \mathrm{mmol} / \mathrm{L}$ & $5.89(2.22)$ & $5.77(1.96)$ & $5.99(2.41)$ & $<0.001$ \\
\hline $\mathrm{TC}, \mathrm{mmol} / \mathrm{L}$ & $5.73(1.22)$ & $5.45(1.10)$ & $5.98(1.26)$ & $<0.001$ \\
\hline $\mathrm{LDL}-\mathrm{C}, \mathrm{mmol} / \mathrm{L}$ & $3.97(1.02)$ & $3.97(0.93)$ & $4.12(1.07)$ & $<0.001$ \\
\hline $\mathrm{HDL}-\mathrm{C}, \mathrm{mmol} / \mathrm{L}$ & $1.08(0.28)$ & $0.99(0.25)$ & $1.15(0.29)$ & $<0.001$ \\
\hline Non-HDL-C, mmol/ L & $4.46(1.20)$ & $4.46(1.10)$ & $4.2(1.25)$ & $<0.001$ \\
\hline TGs, mmol/ L & $1.87(1.33)$ & $1.84(1.35)$ & $1.90(1.31)$ & 0.06 \\
\hline $\mathrm{TC} / \mathrm{HDL}-\mathrm{C}$ & $5.63(1.77)$ & $5.82(1.83)$ & $5.46(1.70)$ & $<0.001$ \\
\hline $\mathrm{TGs} / \mathrm{HDL}-\mathrm{C}$ & $1.81(1.67)$ & $1.95(1.85)$ & $1.70(1.50)$ & $<0.001$ \\
\hline Current smoker, $n(\%)$ & $862(15.6)$ & $727(28.7)$ & $135(4.5)$ & $<0.001$ \\
\hline Low physical activity, n (\%) & $4401(79.8)$ & 2082(82.2) & 2319(77.7) & $<0.001$ \\
\hline Education level, $n$ (\%) & & & & $<0.001$ \\
\hline$\geq 12$ years & 490(8.9) & $368(14.6)$ & $122(4.1)$ & \\
\hline $6-11$ years & 1939(35.2) & $1065(42.1)$ & $874(29.3)$ & \\
\hline$<6$ years & $3082(55.9)$ & 1096(43.3) & 1986(66.6) & \\
\hline Prevalent CVD, $n(\%)$ & $464(8.4)$ & $252(10.0)$ & $212(7.1)$ & $<0.001$ \\
\hline Prevalent CKD, $n(\%)$ & $1617(29.3)$ & 494(19.5) & 1123(37.6) & $<0.001$ \\
\hline Glucose tolerance, $n(\%)$ & & & & 0.003 \\
\hline Normal glucose tolerance & $3022(57.3)$ & $1442(59.5)$ & 1580(55.4) & \\
\hline Prediabetes & $1445(27.4)$ & $647(26.7)$ & $798(28.0)$ & \\
\hline Diabetes & $806(15.3)$ & $333(13.7)$ & $473(16.6)$ & \\
\hline Blood pressure status, $n(\%)$ & & & & $<0.001$ \\
\hline Normotension & 1714(31.1) & $862(34.0)$ & $852(28.5)$ & \\
\hline Prehypertension & 1832(33.2) & $862(34.0)$ & $970(32.5)$ & \\
\hline Hypertension & 1972(35.7) & $808(31.9)$ & 1164(39.0) & \\
\hline Hypertension drug, $n$ (\%) & 769(13.9) & 230(9.1) & $539(18.1)$ & $<0.001$ \\
\hline Lipid lowering drugs, $n$ (\%) & $306(5.5)$ & $83(3.3)$ & $223(7.5)$ & $<0.001$ \\
\hline Diabetes drug, $n$ (\%) & $424(7.7)$ & $152(6.0)$ & $272(9.1)$ & $<0.001$ \\
\hline
\end{tabular}

Data are shown as mean \pm SD for continuous variables ( $p$ value calculated with the $t$ test), $n$ (\%) for categorical variables ( $p$-value according to the chi-squared test) or median (interquartile range) for TGs and TGs/HDL-C ( $p$-value according to the Mann-Whitney $U$ test)

$B M I$ body mass index, WC waist circumference, SBP systolic blood pressure, $D B P$ diastolic blood pressure, $F P G$ fasting plasma glucose, $T C$ total cholesterol, $L D L-C$ low density lipoprotein cholesterol, HDL-C high density lipoprotein cholesterol, TGs triglycerides, CVD cardiovascular disease, CKD chronic kidney disease

during a long term follow-up in an Iranian adult population. Our findings indicate a divergent associations of TC, LDL-C, non-HDL-C, TC/HDL-C, ln-TGs and lnTGs/HDL-C with CVD vs non-CV mortality, with a higher risk for the former and lower risk for the latter. The absence of any interaction between gender and lipid parameters indicated that being male vs female did not affect the impact of lipid parameters on CVD, CV and non-CV mortality events.
Lipid measures and cardiovascular events and CV mortality Cholesterol is a major component of atherosclerosis and CVD as an expression of atherosclerotic processes. There are extensive epidemiological data demonstrating that high blood cholesterol levels increase CVD and CV mortality $[6,11,15,29]$. In our study, $\mathrm{TC} \geq 6.14 \mathrm{mmol} / \mathrm{L}$ was associated with 85 and $43 \%$ increase in CVD and CV mortality, respectively. This finding is in agreement with World Health Organization recommendations 
Table 2 Hazard ratios of lipid measures for predicting first cardiovascular disease events among participants without prevalent CVD $(n=5054) ;$ Tehran Lipid and Glucose Study (TLGS) $(2001-2012)^{a}$

\begin{tabular}{|c|c|c|c|c|c|}
\hline & Model 1 & & & Model 2 & \\
\hline & $\mathrm{SD}(\mathrm{mmol} / \mathrm{L})$ & HR $(95 \%$ Cl) & $p$-value & $\mathrm{HR}(95 \% \mathrm{Cl})$ & $p$-value \\
\hline Men & & & & & \\
\hline TC & 1.10 & $1.34(1.22-1.47)$ & $<0.001$ & $1.3(1.18-1.43)$ & $<0.001$ \\
\hline LDL-C & 0.93 & $1.37(1.25-1.51)$ & $<0.001$ & $1.33(1.2-1.46)$ & $<0.001$ \\
\hline $\mathrm{HDL}-\mathrm{C}$ & 0.25 & $0.88(0.79-0.98)$ & 0.021 & $0.94(0.84-1.04)$ & 0.24 \\
\hline Non-HDL-C & 1.11 & $1.37(1.25-1.5)$ & $<0.001$ & $1.32(1.20-1.45)$ & $<0.001$ \\
\hline In-TGs & 0.57 & $1.20(1.09-1.3)$ & $<0.001$ & $1.1(1.0-1.21)$ & 0.05 \\
\hline $\mathrm{TC} / \mathrm{HDL}-\mathrm{C}$ & 1.85 & $1.10(1.13-1.28)$ & $<0.001$ & $1.17(1.09-1.25)$ & $<0.001$ \\
\hline In-TGs/HDL-C & 0.7 & $1.2(1.1-1.31)$ & $<0.001$ & $1.1(1.0-1.21)$ & 0.056 \\
\hline Women & & & & & \\
\hline TC & 1.24 & $1.30(1.18-1.43)$ & $<0.001$ & $1.21(1.1-1.34)$ & $<0.001$ \\
\hline LDL-C & 1.05 & $1.30(1.19-1.43)$ & $<0.001$ & $1.22(1.11-1.35)$ & $<0.001$ \\
\hline $\mathrm{HDL}-\mathrm{C}$ & 0.29 & 0.87(0.78-0.98) & 0.02 & $0.91(0.82-1.02)$ & 0.11 \\
\hline Non-HDL-C & 1.23 & $1.32(1.20-1.45)$ & $<0.001$ & $1.23(1.12-1.36)$ & $<0.001$ \\
\hline In-TGs & 0.51 & $1.42(1.27-1.58)$ & $<0.001$ & $1.24(1.1-1.4)$ & $<0.001$ \\
\hline TC/HDL-C & 1.69 & $1.33(1.21-1.46)$ & $<0.001$ & $1.26(1.13-1.4)$ & $<0.001$ \\
\hline In-TGs/HDL-C & 0.65 & $1.38(1.23-1.54)$ & $<0.001$ & $1.22(1.09-1.38)$ & 0.001 \\
\hline Total & & & & & \\
\hline TC & 1.21 & $1.33(1.24-1.42)$ & $<0.001$ & $1.26(1.18-1.35)$ & $<0.001$ \\
\hline LDL-C & 1.01 & $1.34(1.26-1.43)$ & $<0.001$ & $1.27(1.19-1.36)$ & $<0.001$ \\
\hline $\mathrm{HDL}-\mathrm{C}$ & 0.28 & $0.88(0.81-0.95)$ & 0.001 & $0.93(0.86-1.007)$ & 0.07 \\
\hline Non-HDL-C & 1.19 & $1.35(1.26-1.44)$ & $<0.001$ & $1.22(1.14-1.30)$ & $<0.001$ \\
\hline In-TGs & 0.53 & $1.28(1.20-1.37)$ & $<0.001$ & $1.15(1.07-1.24)$ & $<0.001$ \\
\hline $\mathrm{TC} / \mathrm{HDL}-\mathrm{C}$ & 1.78 & $1.24(1.18-1.30)$ & $<0.001$ & $1.18(1.12-1.25)$ & $<0.001$ \\
\hline In-TGs/HDL-C & 0.67 & $1.27(1.18-1.36)$ & $<0.001$ & $1.14(1.06-1.23)$ & $<0.001$ \\
\hline
\end{tabular}

Model 1: Lipid profile + gender (for total population); model $2=$ model $1+$ blood pressure status (i.e. normotension, prehypertension and hypertension status), glucose tolerance status (normal glucose tolerance, prediabetes and diabetes), education status, low physical activity, current smoker, lipid lowering drugs and body mass index

a Hazard ratios (HR) indicate the increased risk for every 1-SD increase of each lipid parameter

CVD cardiovascular disease, SD standard deviation, TC total cholesterol, LDL-C low density lipoprotein cholesterol, HDL-C high density lipoprotein cholesterol, In-TGs logarithm-transformed triglycerides, $\mathrm{Cl}$ confidence interval

for CVD risk assessment in low and medium resource countries [30].

In the current study, every 1-SD increment in nonHDL-C and LDL-C was associated with 22 and $27 \%$ increased CVD risk, respectively, findings in line with the results of a meta-analysis regarding markers of cardiovascular risk that demonstrated standardized relative risk for every 1-SD increase in non-HDL-C and LDL-C was 34 and $25 \%$, respectively [3]. Meanwhile, non-HDL$\mathrm{C} \geq 4.09 \mathrm{mmol} / \mathrm{L}$ was associated with $39 \%$ increase in risk of CVD and further $1 \mathrm{mmol} / \mathrm{L}$ increment duplicated the risk. In a recent genetic study including data source from TLGS, several variants with large effects on lipid concentrations and their causal relationship with coronary artery disease (CAD) have been discovered; among them, the non-HDL cholesterol genetic risk score has been associated most strongly with CAD [31].

In the present study, increase in serum TGs and TGs/ HDL-C was associated with increased CVD events, but not $\mathrm{CV}$ mortality. We have previously shown that among Iranian population aged $\geq 50$ years, a 1-SD increment in $\ln$-TGs is significantly associated with more than $27 \%$ increased risk for CHD [18]. A long-standing association exists between elevated TG levels and CVD [32-34]. Epidemiological and genetic evidence supporting impact of raised TG remnant cholesterol or triglyceriderich lipoproteins (TRL) as an additional cause of CVD and all-cause mortality. Nordestgaard et al., acknowledged that TG concentrations of $2-10 \mathrm{mmol} / \mathrm{L}$ create an increased risk of CVD [35]. Moreover, mendelian randomization 
Table 3 Hazard ratios for predicting first cardiovascular disease based on to lipid profile tertiles of participants without prevalent CVD ( $n=5054)$; Tehran Lipid and Glucose Study (TLGS) (2001-2012)

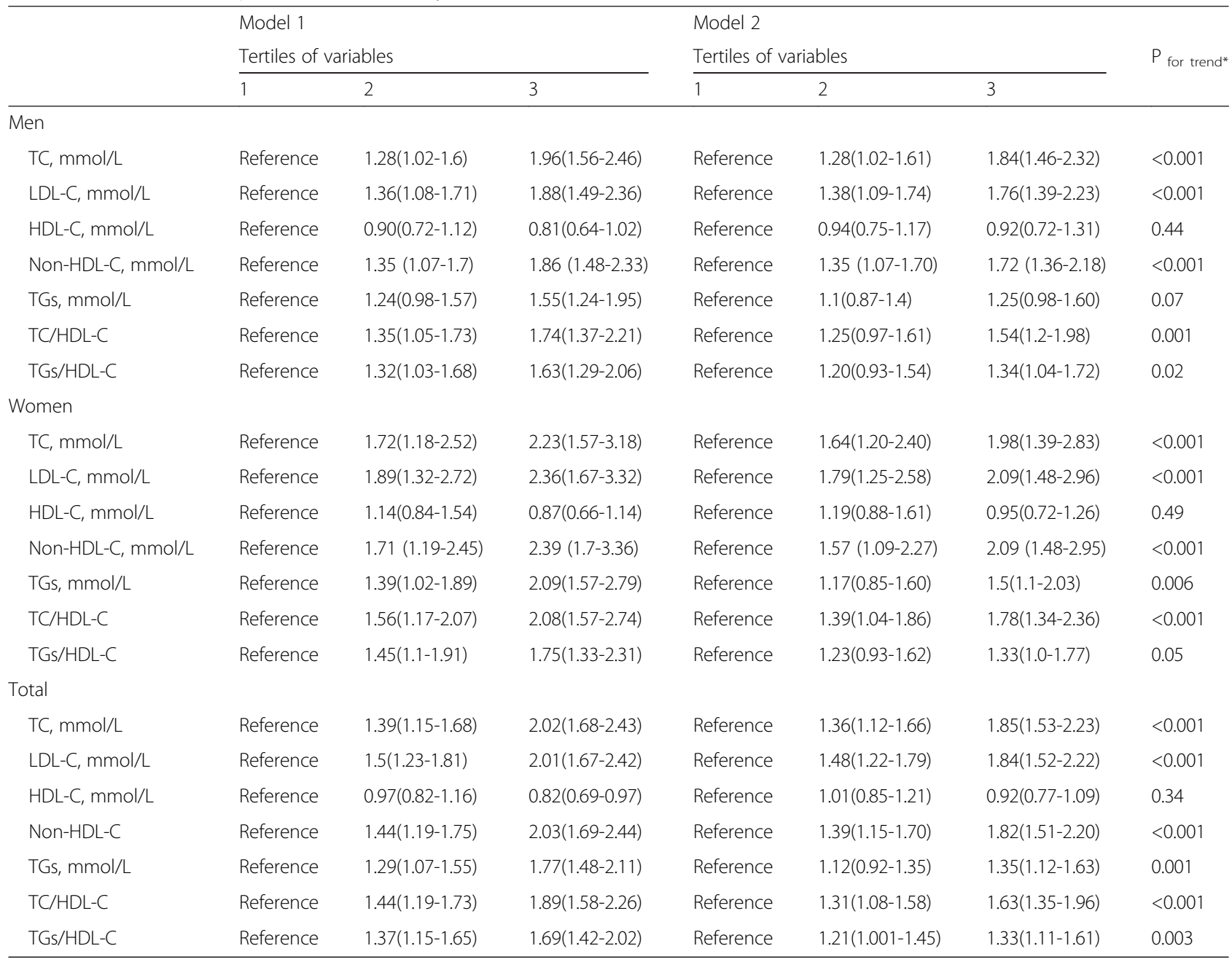

Model 1: lipid profile tertiles + gender (for total population); model $2=$ model $1+$ blood pressure status (i.e. normotension, prehypertension and hypertension status), glucose tolerance status (normal glucose tolerance, prediabetes and diabetes), education status, low physical activity, current smoker, lipid lowering drugs and body mass index

CVD cardiovascular disease, TC total cholesterol, $L D L-C$ low density lipoprotein cholesterol, $H D L-C$ high density lipoprotein cholesterol, $T G s$ triglycerides, $C$ confidence interval. *P-values were calculated using age scale Cox proportional hazards regression models

that can contribute to the evidence or against the causal effect of plasma lipids on atherosclerosis hypothesized causality of TG in CAD risk [36]. Several new variants of angiopoietin-like 4, a lipoprotein lipase inhibitor, leading to decreased serum TG levels, have been associated with protection from CVD $[37,38]$.

There was no significant association between HDL-C and CVD and CV mortality in our study. Different types of HDL-C particles based on their free cholesterol/ phospholipid ratio and availability of apolipoprotein A-1 (apo A-1), exhibit different biological effects including reverse cholesterol transport resulting in different levels of cardio-protection [39]. Jensen et al., report that dysfunctional HDL-C containing apo lipoprotein CIII (apo CIII), stimulates inflammatory and atherogenic responses in cells involved in mortality and atherosclerosis [40]. The level of apo CIII is affected by race/ethnicity [41]. Asians have more dysfunctional apo CIII-containing HDL-C than Western population [42], a condition that could predispose them to higher mortality risk with increasing levels of HDL-C. A recent study conducted on TLGS data revealed increasing HDL-C as a CHD risk factor for premenopausal women [43]. Based on the findings from the Lipoprotein Investigators Collaborative study, some HDL$\mathrm{C}$ subclasses may be responsible for the inverse association of HDL-C with CHD [44]. Moreover, a survey by Rohatgi et al., in a population-based cohort, revealed that although baseline HDL-C level was not associated with CVD, cholesterol efflux capacity, as a novel biomarker of reverse cholesterol transport, was inversely associated with the incidence of CVD [45]. On the other hand, large population-based studies have displayed that subjects who 
Table 4 Hazard ratios of lipid measures for predicting cardiovascular mortality among total participants $(n=5518)$; Tehran Lipid and Glucose Study (TLGS) (2001-2012)

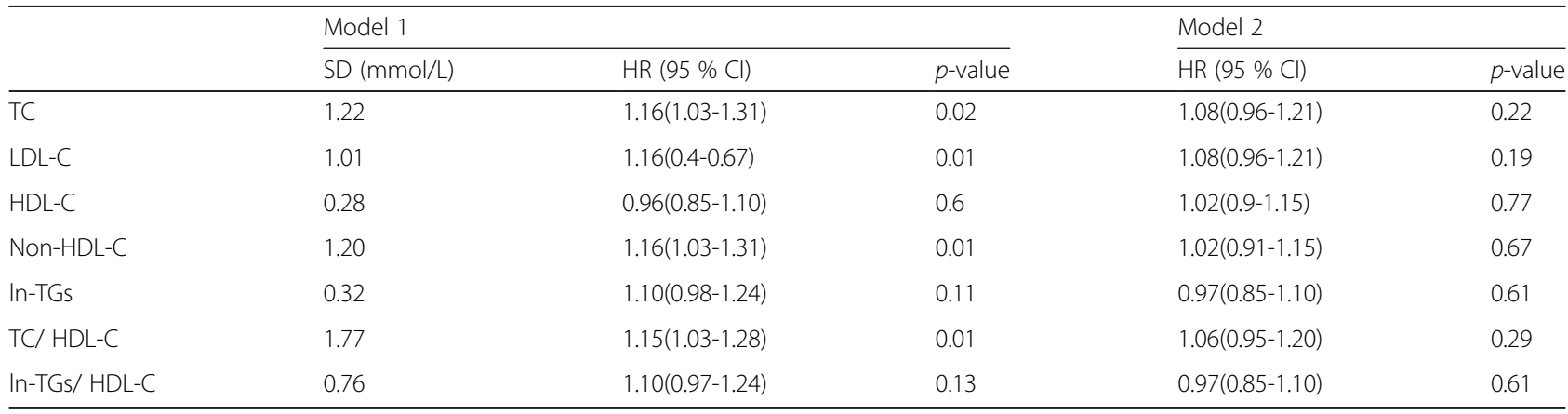

Model 1: lipid profile + gender; model 2 = model 1 + blood pressure status (i.e. normotension, prehypertension and hypertension status), glucose tolerance status (normal glucose tolerance, prediabetes and diabetes), education status, low physical activity, current smoker, lipid lowering drugs, body mass index and prevalent cardiovascular disease

${ }^{a}$ Hazard ratios (HR) indicate the increased risk for a 1-SD increase of each lipid parameter

$S D$ standard deviation, TC total cholesterol, LDL-C low density lipoprotein cholesterol, HDL-C high density lipoprotein cholesterol, In-TGs logarithm-transformed triglycerides, $\mathrm{Cl}$ confidence interval

are carriers of scavenger receptor BI (the major receptor for HDL-C) mutation have significantly increased levels of plasma HDL-C but an increased risk of $\mathrm{CHD}$ (odds ratio = 1.79) [46]. To summarize, for evaluation of the effect of HDL-C on CVD outcomes and mortality events, recent studies emphasize the importance of HDL phenotypes rather than its measured concentration.

\section{Lipid measures and non-cardiovascular mortality}

In the present study, multivariate analysis showed that every 1-SD increment in all lipid measures except HDL$\mathrm{C}$ was associated with 17-27\% decreased risk of non$\mathrm{CV}$ mortality. Several studies have demonstrated the inverse association between lipid parameters and mortality in specific subpopulations including the elderly and those with CKD [13, 47]; however, in the current study the presence of CKD did not remain as a covariate even in the univariate analysis $(P>0.6)$. In a population-based study of individuals aged $\geq 50$ years in Denmark, higher lipoprotein levels (including TC and LDL-C) showed a survival benefit compared with recommended low levels [13]. In the Norwegian Counties Study, it was revealed that the second and third quintiles of TGs (1.10 to $1.93 \mathrm{mmol} / \mathrm{L}$ ) among men were significantly associated with lower risk of all-cause mortality events [48]. Among population of the present study, the inverse relationship between lipid measures and non-CV mortality events did not change after excluding participants with survival $<3$ years and those with BMI $\leq 18.5 \mathrm{~kg} / \mathrm{m}^{2}$ at baseline. The demonstrated inverse association between lipid parameters and non- $\mathrm{CV}$ mortality might be due to the residual effect of other conditions such as malnutrition, inflammation and sarcopenia $[49,50]$ that could not be assessed in our study. Furthermore, socioeconomic status exposing the individuals to a wide range of risk factors for poor health would explain some of the

Table 5 Crude and multivariate adjusted hazard ratios of cardiovascular mortality based on lipid profile tertiles of total participants ( $n=5518) ;$ Tehran Lipid and Glucose Study (TLGS) (2001-2012)

\begin{tabular}{|c|c|c|c|c|c|c|c|}
\hline & Model 1 & & & Model 2 & & & \\
\hline & Tertiles of & oles & & Tertiles of & oles & & \\
\hline & 1 & 2 & 3 & 1 & 2 & 3 & $P_{\text {for trend* }}$ \\
\hline $\mathrm{TC}, \mathrm{mmol} / \mathrm{L}$ & Reference & $1.27(0.93-1.73)$ & $1.52(1.12-2.08)$ & Reference & $1.17(0.84-1.62)$ & $1.43(1.04-1.98)$ & 0.027 \\
\hline $\mathrm{LDL}-\mathrm{C}, \mathrm{mmol} / \mathrm{L}$ & Reference & $1.27(0.93-1.73)$ & $1.42(1.05-1.93)$ & Reference & $1.19(0.86-1.64)$ & $1.29(0.94-1.78)$ & 0.12 \\
\hline $\mathrm{HDL}-\mathrm{C}, \mathrm{mmol} / \mathrm{L}$ & Reference & $0.89(0.66-1.20)$ & $0.89(0.67-1.19)$ & Reference & $0.98(0.72-1.34)$ & $1.06(0.78-1.42)$ & 0.71 \\
\hline Non-HDL-C & Reference & $1.20(0.89-1.63)$ & $1.17(0.87-1.58)$ & Reference & $1.17(0.85-1.61)$ & $1.24(0.9-1.71)$ & 0.19 \\
\hline $\mathrm{TGs}, \mathrm{mmol} / \mathrm{L}$ & Reference & $0.94(0.7-1.26)$ & $1.14(0.86-1.52)$ & Reference & $0.83(0.61-1.13)$ & $0.95(0.69-1.3)$ & 0.77 \\
\hline $\mathrm{TC} / \mathrm{HDL}-\mathrm{C}$ & Reference & $0.96(0.71-1.30)$ & $1.21(0.91-1.61)$ & Reference & $0.93(0.68-1.27)$ & $1.0(0.74-1.36)$ & 0.95 \\
\hline TGs/HDL-C & Reference & $1.23(0.93-1.64)$ & $1.1(0.82-1.5)$ & Reference & $0.96(0.71-1.30)$ & $0.85(0.62-1.18)$ & 0.33 \\
\hline
\end{tabular}

Model 1: lipid profile tertiles + gender; model 2 = model 1 + blood pressure status (i.e. normotension, prehypertension and hypertension status), glucose tolerance status (normal glucose tolerance, prediabetes and diabetes), education status, low physical activity, current smoker, lipid lowering drugs, body mass index and prevalent cardiovascular disease

$T C$ total cholesterol, $L D L-C$ low density lipoprotein cholesterol, $H D L-C$ high density lipoprotein cholesterol, $T G s$ triglycerides, $C l$ confidence interval

${ }^{*} P$-values were calculated using age scale Cox proportional hazards regression models 
Table 6 Hazard ratios of lipid measures for predicting non-cardiovascular mortality among total participants $(n=5518) ;$ Tehran Lipid and Glucose Study (TLGS) (2001-2012)

\begin{tabular}{|c|c|c|c|c|c|}
\hline & \multicolumn{3}{|l|}{ Model 1} & \multicolumn{2}{|l|}{ Model 2} \\
\hline & $\mathrm{SD}(\mathrm{mmol} / \mathrm{L})$ & HR $(95 \% \mathrm{Cl})$ & $p$-value & HR $(95 \%$ Cl) & $p$-value \\
\hline $\mathrm{TC}$ & 1.22 & $0.8(0.70-0.92)$ & 0.001 & $0.76(0.66-0.87)$ & $<0.001$ \\
\hline LDL-C & 1.01 & $0.79(0.69-0.90)$ & $<0.001$ & $0.75(0.66-0.86)$ & $<0.001$ \\
\hline $\mathrm{HDL}-\mathrm{C}$ & 0.28 & $1.08(0.95-1.22)$ & 0.24 & $1.07(0.94-1.21)$ & 0.32 \\
\hline Non-HDL-C & 1.20 & $0.79(0.69-0.90)$ & $<0.001$ & $0.73(0.64-0.84)$ & $<0.001$ \\
\hline In-TGs & 0.32 & $0.89(0.78-1.0)$ & 0.06 & $0.81(0.7-0.93)$ & 0.002 \\
\hline $\mathrm{TC} / \mathrm{HDL}-\mathrm{C}$ & 1.77 & $0.81(0.71-0.94)$ & 0.004 & $0.77(0.67-0.89)$ & 0.001 \\
\hline In-TGs/HDL-C & 0.76 & $0.89(0.79-1.01)$ & 0.076 & $0.83(0.72-0.95)$ & 0.006 \\
\hline
\end{tabular}

Model 1: lipid profile + gender; model 2 = model 1 + blood pressure status (i.e. normotension, prehypertension and hypertension status), glucose tolerance status (normal glucose tolerance, prediabetes and diabetes), education status, low physical activity, current smoker, lipid lowering drugs, body mass index and prevalent cardiovascular disease

${ }^{\mathrm{a}} \mathrm{Hazard}$ ratios indicate the increased risk for a 1-SD increase of each lipid parameter

$S D$ standard deviation, TC total cholesterol, $L D L-C$ low density lipoprotein cholesterol, HDL-C high density lipoprotein cholesterol, In-TGs logarithm-transformed triglycerides, $\mathrm{Cl}$ confidence interval

aforementioned inverse relationship for non-CV mortality $[51,52]$.

\section{Limitations and strengths}

There are several limitations that should be addressed. First, we measured lipid parameters only once at baseline; thus the potential bias resulting from regression dilution could not be ignored. The underestimation of the impact of lipids increased with time interval between baseline and follow-up measurements for TC and TGs, has previously been reported in TLGS cohort [53]. Second, we applied modified Friedwald formula to calculate the level of LDL-C rather than its direct measurement. The collected data are remarkably consistent that LDL particle (LDL-P) number is a better predictor of CV risk compared with the standard measurement of LDL-C concentration; however, these important but costly atherogenic lipid particles were not measured in this large population-based study. On the other hand, still, we need standardization for LDL-P measurements [54]. Third, apo A-1, apo B and lipoprotein(a) (Lp[a]) were not measured in the current study; however, Ingelsson et al. have shown that overall performance of apo B/apo A-1 for prediction of CHD is comparable with that of traditional lipid ratios and results in no incremental utility over TC/HDL-C [55]. Furthermore, a recent metaanalysis including 37 prospective cohorts demonstrated that information on non-HDL-C, apo B, apo A-I and $\mathrm{Lp}$ (a) did not improve CVD prediction provided by simple measurements of TC and HDL-C [56]. Although the Emerging Risk Factors Collaboration displayed continuous associations of $\mathrm{Lp}(\mathrm{a})$ concentration with risk of $\mathrm{CHD}$, they claimed that there is significant variability in measured Lp(a) concentration [57]. Forth, we did not have data regarding inflammatory parameters including C-reactive protein (CRP) and fibrinogen. Yeboah et al.,

Table 7 Crude and multivariate adjusted hazard ratios of non-cardiovascular mortality based on lipid profile tertiles of total participants ( $n=5518) ;$ Tehran Lipid and Glucose Study (TLGS) (2001-2012)

\begin{tabular}{|c|c|c|c|c|c|c|c|}
\hline & Model 1 & & & Model 2 & & & \\
\hline & Tertiles of & & & Tertiles of & oles & & \\
\hline & 1 & 2 & 3 & 1 & 2 & 3 & $P_{\text {for trend* }}$ \\
\hline $\mathrm{TC}, \mathrm{mmol} / \mathrm{L}$ & Reference & $0.73(0.55-0.98)$ & $(0.61-0.45-0.83)$ & Reference & $0.75(0.55-1.01)$ & $0.57(0.42-0.79)$ & 0.001 \\
\hline $\mathrm{LDL}-\mathrm{C}, \mathrm{mmol} / \mathrm{L}$ & Reference & $0.62(0.46-0.83)$ & $0.61(0.45-0.82)$ & Reference & $0.64(0.47-0.87)$ & $0.57(0.42-0.77)$ & $<0.001$ \\
\hline $\mathrm{HDL}-\mathrm{C}, \mathrm{mmol} / \mathrm{L}$ & Reference & $0.86(0.63-1.19)$ & $1.01(0.75-1.34)$ & Reference & $0.97(0.7-1.34)$ & $1.07(0.79-1.45)$ & 0.62 \\
\hline Non-HDL-C & Reference & $0.61(0.45-0.82)$ & $0.63(0.47-0.84)$ & Reference & $0.62(0.46-0.84)$ & $0.58(0.43-0.79)$ & $<0.001$ \\
\hline TGs, mmol/L & Reference & $0.87(0.66-1.15)$ & $0.71(0.52-0.96)$ & Reference & $0.8(0.60-1.08)$ & $0.59(0.42-0.82)$ & 0.002 \\
\hline $\mathrm{TC} / \mathrm{HDL}-\mathrm{C}$ & Reference & $0.78(0.58-1.03)$ & $0.66(0.49-0.90)$ & Reference & $0.77(0.58-1.03)$ & $0.6(0.44-0.83)$ & 0.002 \\
\hline TGs/HDL-C & Reference & $0.91(0.69-1.21)$ & $0.79(0.58-1.06)$ & Reference & $0.82(0.61-1.10)$ & $0.65(0.47-0.91)$ & 0.01 \\
\hline
\end{tabular}

Model 1: lipid profile tertiles + gender; model $2=$ model $1+$ blood pressure status (i.e. normotension, prehypertension and hypertension status), glucose tolerance status (normal glucose tolerance, prediabetes and diabetes), education status, low physical activity, current smoker, lipid lowering drugs, body mass index and prevalent cardiovascular disease

TC total cholesterol, LDL-C low density lipoprotein cholesterol, HDL-C high density lipoprotein cholesterol, TGs triglycerides, $\mathrm{Cl}$ confidence interval

*P-values were calculated using age scale Cox proportional hazards regression models 
have reported improvement in the Framingham Risk Score for prediction of CHD within intermediate-risk participants using additional risk markers; nevertheless, the net reclassification improvement for incident CHD with high-sensitivity CRP was less than coronary artery calcium, brachial flow-mediated dilation, ankle-brachial index, carotid intima-media thickness and family history [58]. On the other hand, during a short term follow-up among Tehranian adult population, we have shown that measurement of CRP has no added value for prediction of CVD, over and above traditional risk factors [59]. Fifth, no information about nutrition transition of our country was available in this period; an issue that might affect the impact of lipid measures on mortality outcomes. Sixth, the statistical power of our study to detect a HR of 1.08 of TC (35\%) and LDL-C (25\%) for CV mortality was low. Lastly, this study was performed in a large sample of urban Tehranian population; hence it is not possible to extrapolate our findings to the other parts of country or Middle-Eastern region.

The strength of our prospective study lies in a reasonable sample size, length of follow-up and direct measurement of the different variables and outcomes rather than self-reported data.; moreover, to the best, it was the first long term prospective and population-based study which investigated the association between different lipid measures and $\mathrm{CV}$ vs non-CV mortality.

\section{Conclusions}

Findings of the present study indicate divergent associations between TC, LDL-C, non-HDL-C, TC/HDL-C, lnTGs and ln TGs/HDL-C and CVD vs non-CV mortality, with a higher risk for the former and lower risk for the latter, during a long term population-based study.

\section{Additional file}

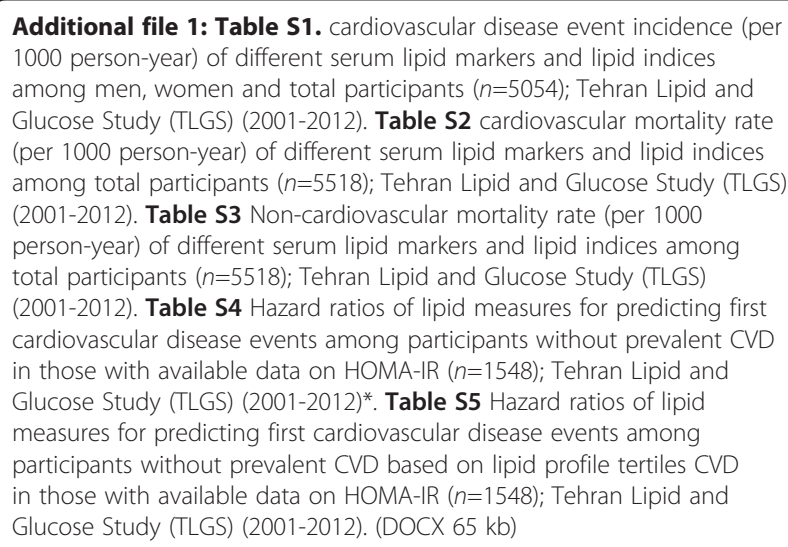

\section{Abbreviations}

2 h-PCPG, 2 h- Post Challenge Plasma Glucose; apo A-1, apolipoprotein A-1; apo B, apolipoprotein B; apo CIII, apolipoprotein CIII; BMI, body mass index;
$C A D$, coronary artery disease; $C H D$, coronary heart disease; CKD, chronic kidney disease; CRP, C-reactive protein; CV, cardiovascular; CVD, cardiovascular disease; DBP, diastolic blood pressure; eGFR, estimated Glomerular Filtration Rate; FPG, fasting plasma glucose; HDL-C, high density lipoprotein-cholesterol; HOMA-IR, homeostatic model assessment-insulin resistance; $H R$, hazard ratio; LDL-C, low density lipoprotein-cholesterol; In, logarithm-transformed; Lp(a), lipoprotein(a); SBP, systolic blood pressure; SD, standard deviation; TC, total cholesterol; TGs, triglycerides; TLGS, tehran lipid and glucose study; TRL, triglyceride-rich lipoproteins; WC, waist circumference.

\section{Acknowledgements}

This article has been extracted from the thesis of Dr Zahra Ghasemzadeh in School of Medicine Shahid Beheshti University of Medical Sciences. We would like to express our appreciation to TLGS participants and research team members also Ms. Zahra Bahadoran for their enthusiastic support and wish to acknowledge Ms. Niloofar Shiva for critical editing of the English grammar and syntax of the manuscript.

Funding

There is no funding

\section{Authors' contributions}

FH, ZG and HA designed and performed the study and wrote the manuscript. SA conducted statistical analysis. SA, MT, DK, MV, SM, VE and FA revised the manuscript critically for important intellectual content. All authors read and approved the final manuscript.

\section{Competing interest}

The authors declare that they have no competing interests.

\section{Ethics approval and consent to participate}

The study protocol was approved by the ethical committee of the Research Institute for Endocrine Sciences, Shahid Beheshti University of Medical

Sciences, Tehran, Iran and all participants provided written informed consent.

\section{Data sharing statement}

Study data has been extracted from the entire Tehran Lipid and Glucose study (TLGS), a longitudinal and population-based prospective study performed on a representative sample of an urban population of Tehran (the capital of Iran). Statistical analyses have been performed with use of Stata (version 12 SE) software and the statistical codes can be made available from the corresponding author on request.

\section{Author details}

'Prevention of Metabolic Disorders Research Center, Research Institute for Endocrine Sciences, Shahid Beheshti University of Medical Sciences, P.O. Box: 19395-476, Tehran, Iran. ${ }^{2}$ Endocrine Research Center, Research Institute for Endocrine Sciences, Shahid Beheshti University of Medical Sciences, Tehran, Iran. ${ }^{3}$ Obesity Research Center, Research Institute for Endocrine Science, Shahid Beheshti University of Medical Sciences, Tehran, Iran.

Received: 9 March 2016 Accepted: 19 June 2016

Published online: 24 June 2016

\section{References}

1. Wong ND. Epidemiological studies of $\mathrm{CHD}$ and the evolution of preventive cardiology. Nat Rev Cardiol. 2014;11:276-89.

2. Hatmi Z, Tahvildari S, Motlag AG, Kashani AS. Prevalence of coronary artery disease risk factors in Iran: a population based survey. BMC Cardiovasc Disord. 2007;7:32.

3. Sniderman AD, Williams K, Contois JH, Monroe HM, McQueen MJ, de Graaf J, et al. A meta-analysis of low-density lipoprotein cholesterol, non-highdensity lipoprotein cholesterol, and apolipoprotein B as markers of cardiovascular risk. Circ Cardiovasc Qual Outcomes. 2011;4:337-45.

4. von Mühlen D, Langer RD, Barrett-Connor E. Sex and time differences in the associations of non-high-density lipoprotein cholesterol versus other lipid and lipoprotein factors in the prediction of cardiovascular death (The Rancho Bernardo Study). Am J Cardiol. 2003:91:1311-5.

5. Liu J, Zeng F-F, Liu Z-M, Zhang C-X, Ling W, Chen Y-M. Effects of blood triglycerides on cardiovascular and all-cause mortality: a systematic review and meta-analysis of 61 prospective studies. Lipids Health Dis. 2013;12:159. 
6. Stamler J, Daviglus ML, Garside DB, Dyer AR, Greenland P, Neaton JD. Relationship of baseline serum cholesterol levels in 3 large cohorts of younger men to long-term coronary, cardiovascular, and all-cause mortality and to longevity. Jama. 2000;284:311-8.

7. Arsenault BJ, Rana JS, Stroes ES, Després J-P, Shah PK, Kastelein JJ, et al. Beyond low-density lipoprotein cholesterol: respective contributions of non-highdensity lipoprotein cholesterol levels, triglycerides, and the total cholesterol/ high-density lipoprotein cholesterol ratio to coronary heart disease risk in apparently healthy men and women. J Am Coll Cardiol. 2009;55:35-41.

8. Rader DJ, Hovingh GK. HDL and cardiovascular disease. Lancet. 2014;384:618-25.

9. Vega GL, Barlow CE, Grundy SM, Leonard D, DeFina LF. Triglyceride-toHigh-Density-Lipoprotein-Cholesterol Ratio Is an Index of Heart Disease Mortality and of Incidence of Type 2 Diabetes Mellitus in Men. J Investig Med. 2014;62:345-9.

10. Hadaegh F, Khalili D, Ghasemi A, Tohidi M, Sheikholeslami F, Azizi F. Triglyceride/HDL-cholesterol ratio is an independent predictor for coronary heart disease in a population of Iranian men. Nutr Metab Cardiovasc Dis. 2009;19:401-8

11. Nomikos T, Panagiotakos D, Georgousopoulou E, Metaxa V, Chrysohoou C, Skoumas I, et al. Hierarchical modelling of blood lipids' profile and 10-year (2002-2012) all cause mortality and incidence of cardiovascular disease: the ATTICA study. Lipids Health Dis. 2015;14:108.

12. Sung K-C, Ryu S, Wild SH, Byrne CD. An increased high-density lipoprotein cholesterol/apolipoprotein Al ratio is associated with increased cardiovascular and all-cause mortality. Heart. 2015;101(7):553-8. doi:10.1136/ heartjnl-2014-306784.

13. Bathum L, Depont Christensen R, Engers Pedersen L, Lyngsie Pedersen P, Larsen J, Nexøe J. Association of lipoprotein levels with mortality in subjects aged 50+ without previous diabetes or cardiovascular disease: A population-based register study. Scand J Prim Health Care. 2013;31:172-80

14. Peters SA, Singhateh Y, Mackay D, Huxley RR, Woodward M. Total cholesterol as a risk factor for coronary heart disease and stroke in women compared with men: A systematic review and meta-analysis. Atheroscler. 2016;248:123-31.

15. Nagasawa S-y, Okamura T, Iso H, Tamakoshi A, Yamada M, Watanabe M, et al Relation between serum total cholesterol level and cardiovascular disease stratified by sex and age group: a pooled analysis of 65594 individuals from 10 cohort studies in Japan. J Am Heart Assoc. 2012;1:e001974.

16. Nizal Sarrafzadegan M, Sadeghi M, Shahram Oveisgharan M, Marshall T. Incidence of cardiovascular diseases in an Iranian population: the Isfahan Cohort Study. Arch Iran Med. 2013;16:138.

17. Gaziano TA, Bitton A, Anand S, Abrahams-Gessel S, Murphy A. Growing epidemic of coronary heart disease in low-and middle-income countries. Curr Probl Cardiol. 2010;35:72-115.

18. Tohidi M, Mohebi R, Cheraghi L, Hajsheikholeslami F, Aref S, Nouri S, et al. Lipid profile components and incident cerebrovascular events versus coronary heart disease; the result of 9 years follow-up in Tehran Lipid and Glucose Study. Clin Biochem. 2013;46:716-21.

19. Azizi F, Ghanbarian A, Momenan AA, Hadaegh F, Mirmiran P, Hedayati M, et al. Prevention of non-communicable disease in a population in nutrition transition: Tehran Lipid and Glucose Study phase II. Trials. 2009;10:5.

20. Hosseini-Esfahani F, Jessri M, Mirmiran P, Bastan S, Azizi F. Adherence to dietary recommendations and risk of metabolic syndrome: Tehran Lipid and Glucose Study. Metabolism. 2010;59:1833-42.

21. Chen $Y$, Zhang $X$, Pan B, Jin $X, Y$ ao $H$, Chen B, et al. Short paper A modified formula for calculating low-density lipoprotein cholesterol values. Lipids Health Dis. 2010;9:52

22. Association AD. Standards of medical care. Diabetes Care. 2016;39:S13-22.

23. Chobanian AV, Bakris GL, Black HR, Cushman WC, Green LA, Izzo JL, et al. Seventh report of the joint national committee on prevention, detection, evaluation, and treatment of high blood pressure. Hypertens. 2003:42:1206-52.

24. IPAQ Research Committee. Guidelines for data processing and analysis of theinternational physical activity questionnaire (IPAQ)-short and long forms. 2005. Available at http://www.ipaq.ki.se/scoring.htm.

25. Levey AS, Coresh J, Bolton K, Culleton B, Harvey KS, Ikizler TA, et al. KDOQI clinical practice guidelines for chronic kidney disease. Am J Kidney Dis. 2002 39(Suppl 1): S1-S264.

26. Gibbons RJ, Abrams J, Chatterjee K, Daley J, Deedwania PC, Douglas JS, et al. ACC/AHA 2002 guideline update for the management of patients with chronic stable angina - summary article: a report of the American college of cardiology/American heart association task force on practice guidelines
(Committee on the Management of Patients With Chronic Stable Angina). J Am Coll Cardiol. 2003;41:159-68.

27. Pencina MJ, Larson MG, D'Agostino RB. Choice of time scale and its effect on significance of predictors in longitudinal studies. Stat Med. 2007;26:1343-59.

28. Yilmaz B, Sahin K, Bilen H, Bahcecioglu IH, Bilir B, Ashraf S, et al. Carotenoids and non-alcoholic fatty liver disease. Hepatobiliary Surg Nutr. 2015;4:161-71.

29. Tamosiunas A, Luksiene D, Baceviciene M, Bernotiene G, Radisauskas R, Malinauskiene $V$, et al. Health factors and risk of all-cause, cardiovascular, and coronary heart disease mortality: findings from the MONICA and HAPIEE studies in Lithuania. PLoS One. 2014;9:e114283.

30. Programme WHOCD, Organization WH. WHO CVD-risk management package for low-and medium-resource settings. Geneva, Switzerland. World Health Organization; 2002.

31. Helgadottir A, Gretarsdottir S, Thorleifsson G, Hjartarson E, Sigurdsson A, Magnusdottir A, et al. Variants with large effects on blood lipids and the role of cholesterol and triglycerides in coronary disease. Nat Genet. 2016;48(6):634-9.

32. Miller M, Stone NJ, Ballantyne C, Bittner V, Criqui MH, Ginsberg HN, et al. Triglycerides and cardiovascular disease a scientific statement from the American Heart Association. Circulation. 2011;123:2292-333.

33. Hokanson JE, Austin MA. Plasma triglyceride level is a risk factor for cardiovascular disease independent of high-density lipoprotein cholesterol level: a metaanalysis of population-based prospective studies. J Cardiovasc Risk. 1996;3:213-9.

34. Murad MH, Hazem A, Coto-Yglesias F, Dzyubak S, Gupta S, Bancos I, et al. The association of hypertriglyceridemia with cardiovascular events and pancreatitis: a systematic review and meta-analysis. BMC Endocr Disord. 2012;12:1.

35. Nordestgaard BG, Varbo A. Triglycerides and cardiovascular disease. Lancet. 2014;384:626-35.

36. Keenan TE, Rader DJ. Genetics of lipid traits and relationship to coronary artery disease. Curr Cardiol Rep. 2013;15:1-10.

37. Folsom AR, Peacock JM, Demerath E, Boerwinkle E. Variation in ANGPTL4 and risk of coronary heart disease: the atherosclerosis risk in communities study. Metab. 2008;57:1591-6.

38. Myocardial IG, Investigators CEC. Coding variation in ANGPTL4, LPL, and SVEP1 and the risk of coronary disease. N Engl J Med. 2016:374:1134.

39. Lund-Katz S, Lyssenko NN, Nickel M, Nguyen D, Chetty PS, Weibel G, et al. Mechanisms responsible for the compositional heterogeneity of nascent high density lipoprotein. J Biol Chem. 2013;288:23150-60.

40. Jensen MK, Rimm EB, Furtado JD, Sacks FM. Apolipoprotein C-III as a potential modulator of the association between $\mathrm{HDL}$-cholesterol and incident coronary heart disease. J Am Heart Assoc. 2012;1. doi: 10.1161/JAHA.111.000232.

41. Foulkes AS, Wohl DA, Frank I, Puleo E, Restine S, Wolfe ML, et al. Associations among race/ethnicity, ApoC-III genotypes, and lipids in HIV-1-infected individuals on antiretroviral therapy. PLoS Med. 2006;3:337.

42. Dodani S, Kaur R, Reddy S, Reed GL, Navab M, George V. Can dysfunctional $\mathrm{HDL}$ explain high coronary artery disease risk in South Asians? Int J Cardiol. 2008;129:125-32.

43. Nazari SSH, Shakiba M, Khalili D, Hadaegh F, Tohidi M, Azizi F. High-density lipoprotein cholesterol, a protective or a risk factor for developing coronary heart disease? Tehran lipid and glucose study. J Clin Lipidol. 2015:9:553-8.

44. Joshi PH, Toth PP, Lirette ST, Griswold ME, Massaro JM, Martin SS, et al. Association of high-density lipoprotein subclasses and incident coronary heart disease: The Jackson Heart and Framingham Offspring Cohort Studies. Eur J Prev Cardiol. 2014;23(1):41-9. 2047487314543890.

45. Rohatgi A, Khera A, Berry JD, Givens EG, Ayers CR, Wedin KE, et al. HDL cholesterol efflux capacity and incident cardiovascular events. New England J Med. 2014;371:2383-93.

46. Zanoni P, Khetarpal SA, Larach DB, Hancock-Cerutti WF, Millar JS, Cuchel M, et al. Rare variant in scavenger receptor BI raises $\mathrm{HDL}$ cholesterol and increases risk of coronary heart disease. Science. 2016;351:1166-71.

47. Kovesdy CP, Anderson JE, Kalantar-Zadeh K. Inverse association between lipid levels and mortality in men with chronic kidney disease who are not yet on dialysis: effects of case mix and the malnutrition-inflammation-cachexia syndrome. J Am Soc Nephrol. 2007;18:304-11.

48. Lindman AS, Veierød M, Tverdal A, Pedersen J, Selmer R. Nonfasting triglycerides and risk of cardiovascular death in men and women from the Norwegian counties study. Eur J Epidemiol. 2010;25:789-98.

49. Lopez AD, Mathers CD, Ezzati M, Jamison DT, Murray CJ. Global and regional burden of disease and risk factors, 2001: systematic analysis of population health data. Lancet. 2006;367:1747-57.

50. Chang SF, Lin PL. Systematic literature review and meta-analysis of the association of sarcopenia with mortality. Worldviews Evid Based Nurs. 2016;13(2):153-62. 
51. Ullits LR, Ejlskov L, Mortensen RN, Hansen SM, Kræmer SR, VardinghusNielsen $\mathrm{H}$, et al. Socioeconomic inequality and mortality-a regional Danish cohort study. BMC Public Health. 2015;15:1.

52. Shohaimi S, Boekholdt MS, Luben R, Wareham NJ, Khaw K-T. Distribution of lipid parameters according to different socio-economic indicators-the EPICNorfolk prospective population study. BMC Public Health. 2014;14:1.

53. Masudi S, Yavari P, Mehrabi Y, Azizi F, Khalili D, Hadaegh F. Underestimating the effect of lipids on cardiovascular events: regression dilution bias in the population-based cohort of Tehran lipid and glucose study. Int J Endocrinol Metab. 2015;13(4):e27528.

54. Master SR, Rader DJ. Beyond LDL cholesterol in assessing cardiovascular risk: apo B or LDL-P? Clin Chem. 2013;59:723-5.

55. Ingelsson E, Schaefer EJ, Contois JH, McNamara JR, Sullivan L, Keyes MJ, et al. Clinical utility of different lipid measures for prediction of coronary heart disease in men and women. Jama. 2007:298:776-85.

56. Used R. Lipid-related markers and cardiovascular disease prediction. Jama. 2012;307:2499-506.

57. Collaboration ERF. Lipoprotein (a) concentration and the risk of coronary heart disease, stroke, and nonvascular mortality. JAMA. 2009;302:412.

58. Yeboah J, McClelland RL, Polonsky TS, Burke GL, Sibley CT, O'Leary D, et al. Comparison of novel risk markers for improvement in cardiovascular risk assessment in intermediate-risk individuals. Jama. 2012:308:788-95.

59. Tohidi M, Hadaegh F, Harati H, Azizi F. C-reactive protein in risk prediction of cardiovascular outcomes: Tehran Lipid and Glucose Study. Int J Cardiol. 2009;132:369-74.

\section{Submit your next manuscript to BioMed Central and we will help you at every step:}

- We accept pre-submission inquiries

- Our selector tool helps you to find the most relevant journal

- We provide round the clock customer support

- Convenient online submission

- Thorough peer review

- Inclusion in PubMed and all major indexing services

- Maximum visibility for your research

Submit your manuscript at www.biomedcentral.com/submit

C Biomed Central 